EPJ Web of Conferences 41, 09009 (2013)

DOI: $10.1051 /$ epjconf/20134109009

(C) Owned by the authors, published by EDP Sciences, 2013

\title{
Sub-cycle switching of a photonic bandstructure via ultrastrong light-matter coupling
}

\author{
J.-M. Ménard ${ }^{1,2}$, M. Porer ${ }^{1,2}$, A. Leitenstorfer ${ }^{1}$, R. Huber ${ }^{1,2}$, R. Degl'Innocenti ${ }^{3}$, S. Zanotto ${ }^{3}$, G. \\ Biasiol $^{4}$, L. Sorba ${ }^{3}$ and A. Tredicucci ${ }^{3}$ \\ ${ }^{1}$ Department of Physics, University of Konstanz, 78457 Konstanz, Germany \\ ${ }^{2}$ Department of Physics, University of Regensburg, 93040 Regensburg, Germany \\ ${ }^{3}$ NEST, Istituto Nanoscienze-CNR and Scuola Normale Superiore, 56127 Pisa, Italy \\ ${ }^{4}$ Laboratorio TASC, CNR-IOM, Area Science Park, 34149 Trieste, Italy
}

\begin{abstract}
Phase-locked multi-terahertz transients map out the full photonic bandstructure of a one-dimensional photonic crystal while a 12 -fs control pulse activates ultrastrong interaction on a sub-cycle time scale with quantized electronic transitions in semiconductor quantum wells. We trace the build-up dynamics of a large vacuum Rabi splitting and observe an unexpected asymmetric formation of the upper and lower polariton bands. The pronounced flattening of the photonic bands causes a slow-down of the group velocity by one order of magnitude on the time scale of the oscillation period of light.
\end{abstract}

Tailoring optical properties of solids on the sub-wavelength scale has opened exciting possibilities to shape the way light interacts with electronic excitations. Recently, nanostructured optical cavities have been used to reach a new regime of ultrastrong light-matter coupling (USC) where embedded quantum emitters absorb and reemit virtual photons at a rate comparable to the frequency of the bare cavity mode [1-4]. Nonadiabatic modulation of ultrastrong interaction was predicted to cause unconventional quantum electrodynamical phenomena, such as the release of correlated photon pairs [5]. Experimentally, phase-sensitive multi- $\mathrm{THz}$ optoelectronics has been exploited to trace ultrafast activation of USC [2]. First proof-of-principle studies, however, have allowed for a limited control of the dispersion of the photon field only, besides requiring complex coupling geometries.

Here, we combine a one-dimensional photonic crystal (PC) with optically switchable intersubband (ISB) resonances of semiconductor quantum wells (QWs) in order to approach full spatial and temporal control of light. Phase-locked multi-terahertz transients trace an asymmetric formation of the lower polariton (LP) and upper polariton (UP) branches on a time scale of the oscillation period of light. A pronounced flattening of the photonic bands causes a slow-down of the group velocity by one order of magnitude [6]. The room-temperature switching device operated in straightforward transmission geometry paves the way towards studies of nonadiabatic quantum electrodynamics (QED) phenomena.

Figure 1a depicts the design of the semiconductor device used in our experiments. A $2 \mu \mathrm{m}$ thick $\mathrm{Al}_{0.95} \mathrm{Ga}_{0.05} \mathrm{As}$ cladding layer followed by a sequence of $50 \mathrm{GaAs} / \mathrm{Al}_{0.33} \mathrm{Ga}_{0.67} \mathrm{As} \mathrm{QWs}$ is epitaxially grown on an undoped GaAs substrate. The QW region supports a planar waveguide mode. A gold grating, with a period of $a=3.6$ or $4.1 \mu \mathrm{m}$, is then thermally evaporated on the sample surface. The purpose of this grating is twofold: it enables surface plasmon propagation within the QW region and

This is an Open Access article distributed under the terms of the Creative Commons Attribution License 2.0, which permits unrestricted use, distribution, and reproduction in any medium, provided the original work is properly cited. 
(a)

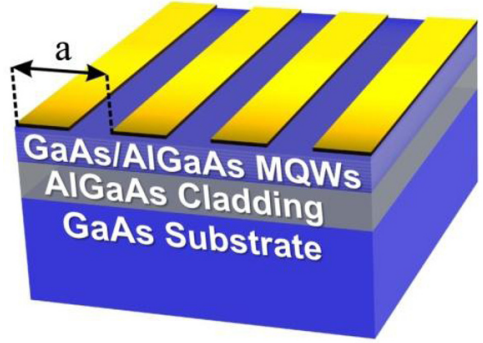

(b)

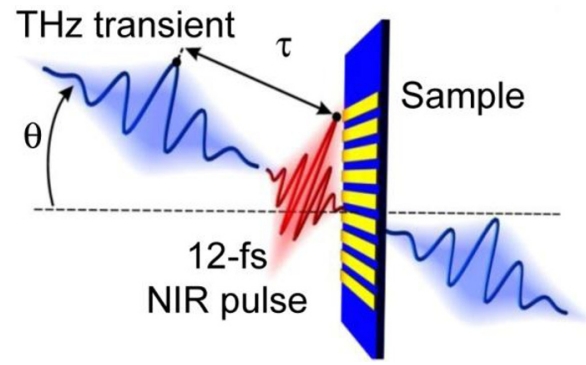

Fig. 1. (a) Schematic of the sample design: 50 repetitions of $8.3 \mathrm{~nm}$ thick GaAs QWs separated by $30 \mathrm{~nm}$ thick $\mathrm{Al}_{0.33} \mathrm{Ga}_{0.67} \mathrm{As}$ barriers grown on top of a $2 \mu \mathrm{m}$ thick $\mathrm{Al}_{0.95} \mathrm{Ga}_{0.05} \mathrm{As}$ cladding layer and an undoped GaAs substrate. A lateral gold grating with period $a=3.6$ or $4.1 \mu \mathrm{m}$ is deposited on top of the heterostructure. (b) Spectrally resolving the transmission of $\mathrm{THz}$ transients allows us to map out the full photonic bandstructure as we vary the angle $\theta$ by rotating the sample. A co-propagating NIR pulse, time delayed by $\tau$, injects electrons in the QWs, thus switching on the USC regime.

folds the photonic dispersion into the first Brillouin zone (BZ). The full bandstructure is mapped out within the first $\mathrm{BZ}$ with the aid of a phase-locked ultrabroadband $\mathrm{THz}$ transient, as we vary its angle of incidence $\theta$ (Figure 1b). Electro-optic sampling (EOS) records both amplitude and phase of the transmitted $\mathrm{THz}$ transient with sub-cycle resolution. A collinear 12-fs near-infrared (NIR) pulse injects electrons into the lowest conduction subband $|1\rangle$ of the QWs and activates light-matter interaction between the optical mode and the transition between $|1\rangle$ and the first excited conduction subband $|2\rangle$. By varying the time delay $\tau$ between the THz and the NIR pulse, we resolve dramatic modifications of the bandstructure occurring faster than a cycle of light.

Eigenmodes of the PC structure are assigned to local transmission peaks which are highlighted by black spheres in Figure 2. The unperturbed sample (Figure 2a) features a dominant photonic mode shifting from a center frequency of $26.7 \mathrm{THz}$ to $32.5 \mathrm{THz}$ as we vary $\theta$ from 0 to $40^{\circ}$. Optical injection of carriers in the sample enables interaction between this bare optical mode and the $|1\rangle-|2\rangle$ ISB transition frequency at $v_{12}=29 \mathrm{THz}$. Figure $2 \mathrm{~b}$ is a snapshot of the bandstructure taken at a delay time of $\tau=1000 \mathrm{ps}$, with a NIR excitation fluence $\Phi=1.6 \mathrm{~mJ} / \mathrm{cm}^{2}$. The dressed photonic mode is now clearly split into LP and UP branches. The minimum separation of $3 \mathrm{THz}$ amounts to more than $10 \%$ of $v_{12}$ and exceeds values demonstrated in semiconductor microcavities
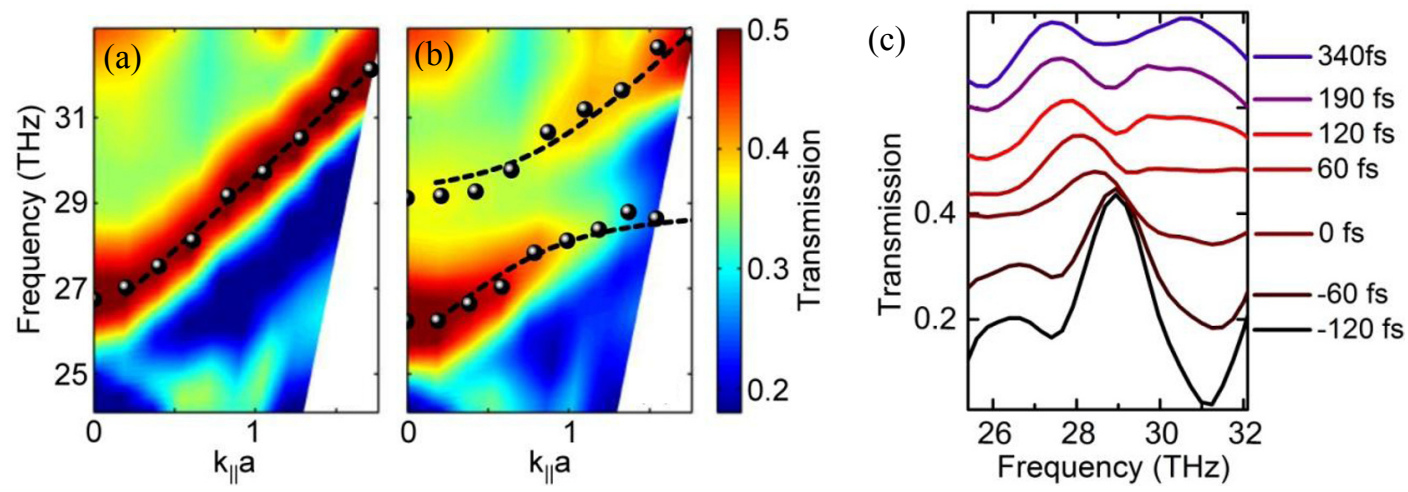

Fig. 2. (a,b) Transmission spectra of a PC structure $(a=4.1 \mu \mathrm{m})$ as a function of $\mathrm{k}_{\|}=\mathrm{k}_{0} \sin (\theta)$. Experimental results (a) in equilibrium and (b) 1000 ps after photo-activation of the ISB transition $\left(\Phi=1.6 \mathrm{~mJ} / \mathrm{cm}^{2}\right)$. The dashed curves highlight maxima of the transmission spectra (black spheres). (c) Transmission spectra of a free standing PC membrane $(a=3.6 \mu \mathrm{m})$ trace the ultrafast formation of polaritons at the anticrossing point $\left(\theta=13^{\circ}\right)$ for selected delay times $\tau$ (on the right) with $\Phi=1.6 \mathrm{~mJ} / \mathrm{cm}^{2}$. 
with static doping [4]. Simultaneously the extreme coupling strength causes a prominent flattening of the bandstructure giving rise to a slow-down of light propagating inside the QW region. A coherent photon population prepared in the bare waveguide can be abruptly transferred into the polariton bands, thus decreasing its group velocity by a factor of up to 13. In this regards, our results compare advantageously with broadband generation of slow light in PCs [7,8]. More strikingly, our scheme provides, for the first time, access to a regime of nonadiabatic slow light generation by enabling sub-cycle modulation of the group velocity via an optically controlled USC.

The ultrafast buildup of light-matter coupling is investigated by probing the anti-crossing point where the bare photonic band and the ISB transition spectrally overlap. Figure 2c shows transmission measurements for selected delay times $\tau$. For negative delay times $(\tau \leq-60 \mathrm{fs})$, the transmission spectra are dominated by a single eigenmode centered at $29 \mathrm{THz}$, characteristic of the unpumped state. When the maxima of the THz and NIR pulses coincide, at $\tau=0$ fs, a nonadiabatic change of the transmission spectrum manifests itself. Instead of the bare eigenmode, two resonances occur, corresponding to the LP and UP branches. Intriguingly, the UP initially exhibits a lower amplitude than the LP, it also remains spectrally much broader. At $\tau=340 \mathrm{fs}$, a maximum splitting of $3.4 \mathrm{THz}$ is reached. At this point, both polariton branches are comparable in intensity and width. The reproducible asymmetric buildup of the polaritons is in stark contrast to the dynamics seen in planar waveguides [2]. Since the difference between both designs is given by the photonic bandstructure, this discrepancy clearly indicates that the folded bandstructure of the PC sets a characteristic dynamics for the formation of USC. Due to energy-time uncertainty, abrupt switching events have to involve a broad spectrum of eigenmodes. Contrary to planar waveguides, this spectrum is strongly structured in the case of a PC. We suggest that the asymmetric buildup arises from a novel scenario of coherent interaction of the ISB transition with multiple photonic bands. A quantitative model of this fascinating new class of ultrafast quantum phenomena is yet missing. Therefore, our observations call for a universal theory treating nonadiabatic quantum electrodynamics and quantum kinetic carrier dynamics, on equal footing.

In conclusion, we demonstrate a nonadiabatic activation of extremely strong plasmon-assisted light-matter interaction in a PC. A collinear configuration allows us to map out the full bandstructure and resolve its dynamics on a time shorter than a single cycle of light. On the femtosecond scale, the bandstructure is dramatically changed featuring an opening of an energy gap as large as $3.4 \mathrm{THz}$. Our approach paves the way towards complete bandstructure engineering in all four dimensions. A fundamentally new area of ultrafast quantum electrodynamical phenomena involving the abrupt creation of white and black hole horizons via slow light as well as vacuum photon emission may be envisaged [9].

1. D. Dini, R. Köhler, A. Tredicucci, G. Biasiol, L. Sorba, Phys. Rev. Lett. 90, 116401 (2003)

2. G. Günter, A. A. Anappara, J. Hees, A. Sell, G. Biasiol, L. Sorba, S. De Liberato, C. Ciuti, A. Tredicucci, A. Leitenstorfer, R. Huber, Nature 458, 178 (2009)

3. Y. Todorov, A. M. Andrews, I. Sagnes, R. Colombelli, P. Klang, G. Strasser, C. Sirtori, Phys. Rev. Lett. 102, 186402 (2009)

4. S. Zanotto, G. Biasiol, R. Degl'Innocenti, L. Sorba, A. Tredicucci, Appl. Phys. Lett. 97, 231123 (2010)

5. C. Ciuti, G. Bastard, I. Carusotto, Phys. Rev. B 72, 115303 (2005)

6. M. Porer, J.-M. Ménard, A. Leitenstorfer, R. Huber, R. Degl'Innocenti, S. Zanotto, G. Biasiol, L. Sorba, A. Tredicucci, Phys. Rev. B 85, 081302(R) (2012)

7. M. D. Settle, R. J. P. Engelen, M. Salib, A. Michaeli, L. Kuipers, T. F. Krauss, Opt. Express 15, 219 (2007)

8. Y. A. Vlasov, M. O’Boyle, H. F. Hamann, and S. J. McNab, Nature 438, 65 (2005)

9. F. Belgiorno, S. L. Cacciatori, M. Clerici, V. Gorini, G. Ortenzi, L. Rizzi, E. Rubino, V. G. Sala, D. Faccio, Phys. Rev. Lett. 105, 203901 (2010) 